\title{
NON-PROPERNESS OF AMENABLE ACTIONS ON GRAPHS WITH INFINITELY MANY ENDS
}

\author{
Soyoung Moon and Alain Valette
}

May 12, 2007

\begin{abstract}
We study amenable actions on graphs having infinitely many ends, giving a generalized answer to Ceccherini's question on groups with infinitely many ends.
\end{abstract}

\section{Statement of the result}

An action of a group $G$ on a set $X$ is amenable if there exists a $G$-invariant mean on $X$, i.e. a map $\mu: 2^{X}=\mathcal{P}(X) \rightarrow[0,1]$ such that $\mu(X)=1, \mu(A \cup B)=\mu(A)+\mu(B)$, for every disjoint subsets $A, B \subseteq X$, and $\mu(g A)=\mu(A), \forall g \in G, \forall A \subseteq X$.

An isometric action of a group $G$ on a metric space $(X, d)$ is proper if for some $x_{0} \in X$, and every $R>0$, the set $\left\{g \in G \mid d\left(x_{0}, g x_{0}\right) \leq R\right\}$ is finite.

The aim of this note is to give a short proof of the following result:

Theorem 1. Let $X=(V, E)$ be a locally finite graph with infinitely many ends. Let $\bar{X}=V \cup \partial X$ be the end compactification. Let $G$ be a group of automorphisms of $X$. Assume that the action of $G$ on $V$ is amenable and there exists $x_{0} \in V$ such that the orbit $G x_{0}$ is dense in $\bar{X}$. Then there is a unique $G$-fixed end in $\partial X$, and the action of $G$ (as a discrete group) on $V$ is not proper.

A deep result of Stallings [4] says that $G$ has infinitely many ends if and only if $G$ is an amalgamated free product $\Gamma_{1} *_{A} \Gamma_{2}$ or $H N N$-extension $H N N(\Gamma, A, \varphi)$ with $A$ finite (with $\min \left\{\left[\Gamma_{1}: A\right],\left[\Gamma_{2}: A\right]\right\} \geq 2$, not both 2 , in the amalgamated product case; and $\min \{[\Gamma: A],[\Gamma: \varphi(A)]\} \geq 2$, not both 2 , in the $H N N$ case). In particular, if $G$ has infinitely many ends, it contains non-abelian free subgroups, hence is non amenable. Tullio Ceccherini-Silberstein asked whether non-amenability of $G$ could be proved without appealing to Stallings' theorem. Since a finitely generated group $G$ with infinitely many ends acts properly and transitively on its Cayley graph, our result shows that $G$ is not amenable.

\section{Remarks}

1. The density assumption of Theorem 1 is satisfied when $G$ has finitely many orbits in $V$. This assumption is necessary; for example the action of $\mathbb{Z}$ on $\mathbb{F}_{2}=\langle a, b\rangle$ defined by $n \cdot g=a^{n} g, \forall n \in \mathbb{Z}, \forall g \in \mathbb{F}_{2}$ is amenable and proper. 
2. Except for the non properness statement, our result is contained in a result of Woess (see Theorem 1 in [6]): if $X=(V, E)$ is a locally finite graph and $G$ admits an amenable action on $V$, then either $G$ fixes a nonempty finite subset of $V$, or $G$ fixes an end of $X$, or $G$ fixes a unique pair of ends which are the fixed points of some hyperbolic element in $G$.

3. There are results on strong isoperimetric inequalities for graphs with infinitely many ends satisfying extra conditions (see Theorem 10.10 in [8]): these give alternative answers to Ceccherini's question.

4. A stronger question is to prove without appealing to Stallings' result that a finitely generated group with infinitely many ends, contains a free group on two generators. Such constructions can be found in the work of Woess (Theorem 3 in [7]), Karlsson and Noskov (Proposition 3 in [3]), and Karlsson (Theorem 1 in [2]).

5. For a finitely generated group with infinitely many ends, Abels shows, using Stallings' theorem, that for $G$ a finitely generated group with infinitely many ends, the compact set of ends is actually a minimal $G$-space (Theorem 1 in [1]). This is false for compactly generated, non discrete groups. Abels indeed gives the example of the group of affine mappings $(x \mapsto a x+b)$ over $\mathbb{Q}_{p}$. This group $G$ is $H N N(K, K, \varphi)$, where $K$ is the group of affine mappings over $\mathbb{Z}_{p}$ and $\varphi: K \rightarrow K$ is given by $(x \mapsto a x+b) \mapsto(x \mapsto a x+p b)$. So $G$ has infinitely many ends, but has a unique fixed point on its space of ends ${ }^{1}$, which is therefore not $G$-minimal.

Acknowledgments We thank T. Ceccherini-Silberstein for suggesting the question, F. Krieger for pointing out a mistake in the previous version, and A. Karlsson for recommending useful references.

\section{Proof of the theorem}

Let $X$ be a countable, discrete set. A compactification of $X$ is a compact space $\bar{X}=X \cup \partial X$ in which $X$ is an open dense subset. If $G$ is a group of permutations of $X$, we say that $\bar{X}$ is a $G$-compactification if the action of $G$ on $X$ extends to an action of $G$ on $\bar{X}$ by homeomorphisms. When $X$ is a locally finite graph (identified with its set of vertices), we will take for $\partial X$ the set of ends of $X$. In this case, we say that $\bar{X}=X \cup \partial X$ is the end-compactification of $X$ (it is an Aut $(X)$-compactification).

Lemma 2. Assume that $G$ admits an amenable action without finite orbit, on a countable set $X$. Let $\mu$ be $G$-invariant mean on $X$. Let $\bar{X}$ be a $G$-compactification of $X$. Then for every subset $A$ of $X$ with $\mu(A)=1$, the set $\left(\bigcap_{g \in G} \overline{g A}\right) \cap \partial X$ is not empty.

Proof. By compactness of $\partial X$, it is enough to show that the family $(\overline{g A} \cap \partial X)_{g \in G}$ has the finite intersection property. For $g_{1}, \ldots, g_{n} \in G$, we have $\mu\left(\bigcap_{i=1}^{n} g_{i} A\right)=1$, while $\mu(F)=0$ for every finite subset $F \subset X$ since $G$ has no finite orbit. So $\bigcap_{i=1}^{n} g_{i} A$ is infinite. Therefore $\left(\overline{\bigcap_{i=1}^{n} g_{i} A}\right) \cap \partial X \neq \emptyset$. A fortiori $\bigcap_{i=1}^{n}\left(\overline{g_{i} A} \cap \partial X\right) \neq \emptyset$.

\footnotetext{
${ }^{1}$ This can be seen directly; it also follows from our result, as $G$ is amenable as a discrete group.
} 
The proof of Theorem 1 will follow from the four claims below:

Claim 1. Let $K$ be a finite, connected subgraph of $X$. Let $A$ be an unbounded connected component of $X \backslash K$. Then $g K \subset A$ for infinitely many $g$ 's in $G$.

By the assumption, any $G$-orbit in $X$ has infinite intersection with $A$ (indeed, the assumption implies that $G x$ is dense in $\bar{X}$ for every vertex $x$ in $V$ since $G$ acts by isometries on $X$; therefore the intersection of $G x$ and $A$ is infinite since $\bar{A}$ is a neighborhood of all ends contained in it). So for $x \in K$, one finds a sequence $\left(g_{n}\right)_{n \geq 1}$ in $G$ such that $g_{n} x$ 's are pairwise distinct vertices in $A$. Since $d\left(g_{n} x, x\right) \rightarrow \infty$ for $n \rightarrow \infty$, we have $g_{n} K \cap K=\emptyset$ for $n$ sufficiently large. Then $g_{n} K$ is a connected subset of $X \backslash K$, and $g_{n} K \cap A \neq \emptyset$. By maximality of $A$ among connected subsets of $X \backslash K$, this implies $g_{n} K \subset A$.

If $K$ is a finite connected subgraph of $X$, we will say that $K$ is good if every connected component of $X \backslash K$ is infinite. Let $K$ be an arbitrary finite connected subgraph of $X$. Denote by $\widehat{K}$ the union of $K$ and the finite connected components of $X \backslash K$; then $\widehat{K}$ is a good subgraph of $X$.

Claim 2. Let $K$ be a good subgraph of $X$, such that $X \backslash K$ has at least 3 connected components. Let $\mu$ be $G$-invariant mean on $V$. Then there exists a unique connected component $C_{K}$ of $X \backslash K$ such that $\mu\left(C_{K}\right)=1$.

Indeed, let $A_{1}, \ldots, A_{n}$ be the connected components of $X \backslash K$ with $n \geq 3$. Without loss of generality, we may assume that $\mu\left(A_{1}\right) \leq \mu\left(A_{i}\right), \forall i \in\{1, \ldots, n\}$.

By claim 1, we can find $h \in A_{1}$ such that $h K \cap K=\emptyset$ and $h K \subset A_{1}$. Since $h A_{1}, \ldots, h A_{n}$ are the connected components of $X \backslash h K$, and $K$ is connected, there exists a unique $k \in\{1, \ldots, n\}$ such that $K \subset h A_{k}$, so that $h A_{i} \subset A_{1}, \forall i \neq k$. Hence $\bigsqcup_{i \neq k} h A_{i} \subset A_{1}$ (see figure 1).

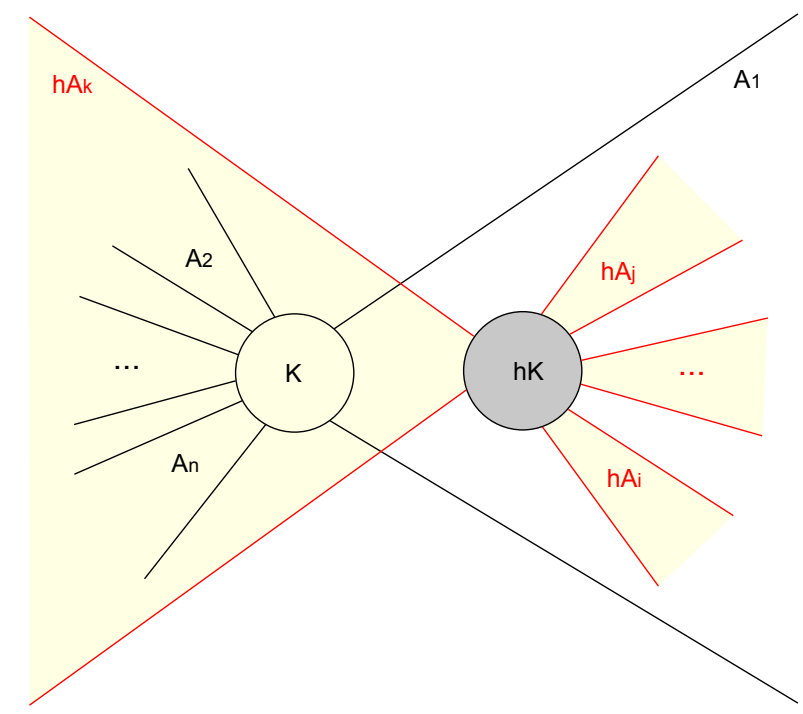

Figure 1. 
Then by minimality of $\mu\left(A_{1}\right)$,

$$
(n-1) \mu\left(A_{1}\right) \leq \sum_{i \neq k} \mu\left(A_{i}\right)=\sum_{i \neq k} \mu\left(h A_{i}\right)=\mu\left(\bigsqcup_{i \neq k} h A_{i}\right) \leq \mu\left(A_{1}\right) .
$$

Hence $\mu\left(A_{1}\right)=0$ since $n \geq 3$, and $\mu\left(A_{i}\right)=0, \forall i \neq k$. Since $\mu$ is zero on finite subsets of $X$, we have $1=\mu(X)=\mu\left(K \cup \bigsqcup_{j=1}^{n} A_{j}\right)=\mu\left(A_{k}\right)$. We set $A_{k}=C_{K}$.

Let $x_{0}$ be a base-vertex in $V$. Denote by $B_{N}$ the ball of radius $N$ centered at $x_{0}$. Let $N_{0}$ be such that, for $N \geq N_{0}$, the complement $X \backslash \widehat{B_{N}}$ has at least 3 connected components. Set

$$
D_{N}=\left(\bigcap_{g \in G} \overline{g C_{\overparen{B_{N}}}}\right) \cap \partial X
$$

By Lemma $2, D_{N} \neq \emptyset$, and $\left(D_{N}\right)_{N \geq N_{0}}$ form a decreasing family of closed non-empty subsets of $\partial X$. So by compactness, $E=\bigcap_{N \geq N_{0}} D_{N}$ is non-empty, and obviously $G$-invariant.

Claim 3. The set $E$ is reduced to one point, and $G$ has no other fixed point in $\partial X$.

Indeed, if $w \in E$ and $w^{\prime} \in \partial X$ with $w \neq w^{\prime}$, then for $N$ large enough $w$ and $w^{\prime}$ are not in the same closure of connected component of $X \backslash \widehat{B_{N}}$. So $w \in \overline{C_{\overparen{B_{N}}}}$ and $w^{\prime} \notin \overline{C_{\overparen{B_{N}}}}$, which means $w^{\prime} \notin E$.

Let us show that $g w^{\prime} \neq w^{\prime}$ for a suitable $g \in G$. Recall (see e.g. Theorem 4 and 9 in [5]) that an automorphism $h \in A u t(X)$ is of exactly one of 3 possible types:

- elliptic, if $h$ stabilizes some finite subset of $V$.

- parabolic, if $h$ is non-elliptic and fixes exactly one end.

- hyperbolic, if $h$ is non-elliptic and fixes exactly two ends.

Let $A^{\prime} \neq C_{\widehat{B_{N}}}$ be a connected component of $X \backslash \widehat{B_{N}}$ with $w^{\prime} \in \overline{A^{\prime}}$. Let $A$ be a connected component of $X \backslash \widehat{B_{N}}$ distinct from $A^{\prime}$ and $C_{\widehat{B_{N}}}$. By claim 1, we can find $g \in G$ such that $g B_{N} \subset A$. All connected components of $X \backslash \widehat{B_{N}}$ will be mapped into $A$ by $g$, except one. This exceptional connected component is necessarily $C_{\widehat{B_{N}}}$ because $\mu\left(C_{\widehat{B_{N}}}\right)=1$ and $\mu$ is $G$-invariant. In particular, $g A \subset A$, and this inclusion is strict. So $g^{m} A \subset A, \forall m \geq 1$. The sequence $g^{m} x_{0}$ possesses a subsequence $g^{m_{k}} x_{0}$ which converges to an end $\xi$ in $\bar{A}$. It is obvious that $g$ fixes $\xi$; therefore $g$ is hyperbolic fixing exactly $\xi$ and $w$. In particular, $g w^{\prime} \neq w^{\prime}$, as was to be shown.

Claim 4. The action of $G$ (endowed with the discrete topology) on $V$ is not proper.

The proof is inspired by a nice observation due to Karlsson and Noskov (Proposition 4 in [3]; see also Proposition 5 in [2]). As in claim 3, we can find $h \in G$ such that $h^{m} A^{\prime} \subset A^{\prime}, \forall m \geq 1$ so that $h$ is hyperbolic and fixes exactly one end $\eta$ in $\overline{A^{\prime}}$, apart from $w$. With the same $g$ as in Claim 3, let $y_{n}=h^{n} g h^{-n}$. We claim that $y_{n} \neq y_{m}, \forall n \neq m$. Suppose by contradiction that there is $n \neq m$ such that $h^{n} g h^{-n}=h^{m} g h^{-m}$; so there exists $k \neq 0$ such that $h^{k} g=g h^{k}$. Then 
$h^{k} g \eta=g h^{k} \eta=g \eta$ since $h$ fixes $\eta$. Since $h^{k}$ fixes the same ends as $h, g \eta$ has to be $\eta$ or $w$. But this is not possible since $\eta, \xi$ and $w$ are all distinct.

Now, it remains for us to prove that the set $\left\{y_{n} x_{0}: n \in \mathbb{N}\right\}$ is bounded. Indeed, for $\gamma$ a hyperbolic automorphism, let $\ell(\gamma)=: \min \left\{d\left(\gamma^{k} v, v\right): k \in \mathbb{Z} \backslash\{0\}, v \in V\right\}$ be the translation length of $\gamma$, and let $L_{\gamma}=:\{v \in V: d(\gamma v, v)=\ell(\gamma)\}$ be the axis of $\gamma$ (this is a line in $X$ ). We will use one more result of Halin [5]: the end $w$, being a fixed end of some hyperbolic automorphism, is thin, i.e. for $N \gg 1$ the set $C_{B_{N}}$ contains finitely many disjoint rays. As a consequence, the rays $L_{h} \cap C_{\widehat{B_{N}}}$ and $L_{g} \cap C_{\widehat{B_{N}}}$ stay within finite distance, i.e. there exists $R>0$ such that, for every $x \in L_{h} \cap C_{\widehat{B_{N}}}$, one can find $x^{\prime} \in L_{g} \cap C_{\widehat{B_{N}}}$ with $d\left(x, x^{\prime}\right) \leq R$.

To prove that $\left\{y_{n} x_{0}: n \in \mathbb{N}\right\}$ is bounded, we may clearly assume that $x_{0} \in$ $L_{h}$. For $n$ large enough, we have $h^{-n} x_{0} \in C_{B_{N}}$, so we can find $x_{n} \in L_{g}$ with $d\left(h^{-n} x_{0}, x_{n}\right) \leq R$. Then,

$$
\begin{aligned}
d\left(y_{n} x_{0}, x_{0}\right) & =d\left(g h^{-n} x_{0}, h^{-n} x_{0}\right) \\
& \leq d\left(g h^{-n} x_{0}, g x_{n}\right)+d\left(g x_{n}, x_{n}\right)+d\left(x_{n}, h^{-n} x_{0}\right) \\
& \leq 2 R+\ell(g)
\end{aligned}
$$

this concludes the proof.

\section{References}

[1] H. Abels, On a problem of Freudenthal's, Compositio Mathematica, 35 (1977), no. $1,39-47$.

[2] A. KARLSson, Free subgroups of groups with nontrivial Floyd boundary, Comm. Algebra, 31 (2003) 5361-5376.

[3] A. Karlsson and Guennadi A. Noskov, Some groups having only elementary actions on metric spaces with hyperbolic boundaries, Geom. Dedicata, 104 (2004) 119-137.

[4] J. R. Stallings, On torsion-free groups with infinitely many ends, Ann. of Math. (2) 88 (1968) 312-334.

[5] R. HALIN, Automorphisms and endomorphisms of infinite locally finite graphs, Abh. Math. Sem. Univ. Hamburg. 39 (1973), 251-283.

[6] W. Woess, Amenable group actions on infinite graphs, Math. Ann. 284, (1989), 251-265.

[7] W. Woess, Fixed sets and free subgroups of groups acting on metric spaces, Math. Z. 214 (1993), no. 3, 425-439.

[8] W. Woess, Random walks on infinite graphs and groups, Cambridge tracts in mathematics 138, Cambridge university press, 2000.

Institut de Mathématiques - Université de Neuchâtel

11, Rue Emile Argand - BP 158, 2009 Neuchâtel - Switzerland

E-mail: so.moon@unine.ch, alain.valette@unine.ch 